\title{
Dueling lasers! A comparative analysis of two different sodium laser technologies on sky
}

Eduardo Marin, Gaetano Sivo, Vincent Garrel, Morten Andersen, Francois Rigaut, et al.

Eduardo Marin, Gaetano Sivo, Vincent Garrel, Morten Andersen, Francois Rigaut, Marcos van Dam, Benoit Neichel, Cristian Moreno, Emmanuel Chirre, Allen Hankla, Rodrigo Carrasco, Constanza Araujo, Gabriel Perez, Pablo Diaz, Angelic Ebbers, Paul Collins, Vicente Vergara, Joy Chavez, Lindsay Magill, Ariel Lopez, Michiel van der Hoeven, Rene Rutten, Paul Hirst, Manuel Lazo, "Dueling lasers! A comparative analysis of two different sodium laser technologies on sky," Proc. SPIE 10703, Adaptive Optics Systems VI, 107033N (11 July 2018); doi: 10.1117/12.2312768

Event: SPIE Astronomical Telescopes + Instrumentation, 2018, Austin, Texas, United States 


\title{
Dueling lasers! A comparative analysis of two different sodium laser technologies on sky
}

Eduardo Marin*a, Gaetano Sivo ${ }^{\mathrm{a}}$, Vincent Garrel ${ }^{\mathrm{d}}$, Morten Andersen ${ }^{\mathrm{a}}$, Francois Rigaut ${ }^{\mathrm{b}}$, Marcos van Dam $^{\mathrm{c}}$, Benoit Neichel ${ }^{\mathrm{e}}$, Cristian Moreno ${ }^{\mathrm{a}}$, Emmanuel Chirre ${ }^{\mathrm{a}}$, Allen Hankla ${ }^{\mathrm{f}}$, Rodrigo Carrasco ${ }^{\mathrm{a}}$, Constanza Araujo ${ }^{\mathrm{g}}$, Gabriel Perez ${ }^{\mathrm{a}}$, Pablo Diaz ${ }^{\mathrm{a}}$, Angelic Ebbers ${ }^{\mathrm{a}}$, Paul Collins ${ }^{\mathrm{a}}$, Vicente Vergara, Joy Chavez ${ }^{\mathrm{a}}$, Lindsay Magilla, Ariel Lopez ${ }^{\mathrm{a}}$, Michiel van der Hoeven ${ }^{\mathrm{a}}$, Rene Rutten ${ }^{\mathrm{a}}$, Paul Hirst ${ }^{\mathrm{a}}$, Manuel Lazo ${ }^{\text {a }}$

${ }^{\mathrm{a}}$ Gemini Observatory, La Serena, Chile; ${ }^{\mathrm{b}}$ Research School of Astronomy and Astrophysics, Austalian National University, Australia; ${ }^{\mathrm{c}}$ Flat Wavefronts, New Zealand; ${ }^{\mathrm{d}}$ Max Plank Insitutu fuer extraterrestrische, Germany; ${ }^{\mathrm{e}}$ Laboratoire d'Astrophysique de Marseille, France; ${ }^{\mathrm{f}}$ Peak to Peak Charter School, Lafayette, Colorado, USA; ${ }^{\mathrm{g}}$ Large Synoptic Survery Telescope, USA

\begin{abstract}
Sodium guide star technologies for Adaptive Optics (AO) have been around for over 20 years. During this time, the technologies for the lasers used to excite the mesospheric sodium have been in constant development, with the goals being not only to excite as much sodium as possible, but to do so efficiently, while producing a round guide star, and while offering a reliable facility. The first lasers in use were dye lasers with a liquid gain medium, while these lasers were able to produce sodium guide stars, the liquid dye used was toxic and flammable. The second generation of guide star lasers used sum-frequency-mixed solid-state lasers. These lasers provided excellent return but were notoriously difficult to calibrate and maintain, requiring a full-time laser engineer on staff. The current third generation of sodium guide star lasers use Raman fiber amplification to generate a laser that is very efficient at exciting sodium with a good spot profile and offer a high degree of reliability. The Gemini South observatory for the last few years has been in the process of obtaining one of these third-generation lasers, a Toptica Sodium Star 20/2 while maintaining its secondgeneration Lockheed Martin Coherent Technologies (LMCT) 50W CW Mode-locked laser. In October of 2017 successful on-sky commissioning of the Toptica laser was executed while the LMCT laser was still active and in operations. During the course of the commissioning run both lasers were used on sky in close in time in possible. We present a comparative study of the performance of each laser.
\end{abstract}

Keywords: AO, LGS

\section{INTRODUCTION}

Adaptive Optics (AO) is a technique used in astronomy to compensate for the induced aberrations in incoming wave fronts by the earth atmosphere. The compensation is done by an adaptive element normally a deformable mirror in real time and enable diffraction limited images on large ground based telescopes that would otherwise be impossible. In order for $\mathrm{AO}$ systems to work a bright source is needed to be able to measure the atmospheric turbulence in real time. The bright source used in the first AO systems were bright Natural Guide Stars (NGS). While NGS are easy to use as the require only a high-speed weave front sensor, they are not always in the location of an object of astronomical interest and thus the use of NGS leads to a very small amount of the sky that can be observed with AO. In order to increase the sky coverage the idea arose to create an artificial guide star (Foy \& Labeyrie, 1985) ${ }^{[1]}$. The first sodium guide star was created on the summit of Mauna Kea Hawaii in 1987 (Thompson and Gardner, $1987)^{[2]}$. Since that time there have been serval generations of sodium guide star lasers. With each new generation comes new advancements in photon return and in operational stability. At the Gemini South observatory as part of the Multi-Conjugate Adaptive Optics System GeMS, two different generations of sodium laser technologies have been used.

*emarin@gemini.edu; $\quad$ phone $\quad 56 \quad 51 \quad 2205-648 ; \quad$ fax $\quad 56 \quad 51 \quad 2205-684 ; \quad$ gemini.edu 
For a full review of GeMS design and commissioning see Rigaut et al. (2014) ${ }^{[3]}$ and Neichel et al. (2014) ${ }^{[4]}$. During the upgrade from one generation to the next we had the unique opportunity to use both lasers on sky and compare their performance. Below we give a brief history of guide star laser technologies and then detail our results on sky.

\subsection{First-generation guide star lasers (Dye Lasers)}

The first generations of lasers used in astronomy were liquid dye lasers. The first laser used by Thompson and Gardner

${ }^{[3]}$ was a dye laser that had been used in LIDAR measurements. This was a pulsed laser and its design has been continued to be used at different LIDAR observatories. Much more powerful dye lasers were later built in the 1990s, with the $1.1 \mathrm{~kW}$ Lawrence Livermore National Laboratory (LLNL) Atomic Vapor Laser Isotope Separation (AVLSI) dye laser producing the brightest ever sodium guide star (Avicola et al., 1994) ${ }^{[5]}$. Leading to a facility sodium guide star laser at the Lick observatory (Friedman, et al. 1995) ${ }^{[6]}$ and also leading to the facility guide star laser at the Keck observatory (Friedman, et al., 1997) ${ }^{[7]}$.

At the same time as the development of the pulsed dye lasers, Continuous-Wave $(\mathrm{CW})$ dye lasers were also being developed. The most well knows in the PARSEC CW dye laser used at the European Southern Observatory (ESO) (Rabien, 2003) ${ }^{[8]}$, which produced the fires sodium LGS in Chile (Banaccini 2006) ${ }^{[9]}$. Dye lasers while used as faculties at multiple sites had issues as the dyes used are often flammable and carcinogenic.

\subsection{Second-generation guide star lasers (Solid State)}

Solid state laser technology began to take off in the 1980s and 1990s and offered an alternative to dye lasers. There distinct technologies where developed. Macro-Micro-Pulsed Sun-Frequency Lasers, Continuous-Wave Sum-Frequency Lasers, and Continuous-Wave Mode-Locked Lasers. The last generation Macro-Micro-Pulsed Sum-Frequency laser developed by The University of Chicago based on the designs of a Massachusetts Institute of Technology/Lincoln Lab (MIT/LL) lasers (Jeys, 1991) ${ }^{[10]}$ and was used on the Palomar 5-meter Hale Telescope (Dekany, et al 2005) ${ }^{[11]}$. The second type of solid state lasers were developed for use at the Starfire Optical Range(SOR), with the final 50 W version being used on sky in 2005 (Denman et al., 2005) ${ }^{[12]}$. The SOR also developed a $20 \mathrm{~W}$ laser and had the opportunity to use both the $50 \mathrm{~W}$ and $20 \mathrm{~W}$ laser on sky with one locked on the sodium D2a line and the other on the D2b, this experiment showed that pumping both sodium lines resulted in a higher sodium return (Drummond, 2007) ${ }^{[13]}$. Lastly the CW ModeLocked lasers were developed at the Gemini, Keck, and Subaru Observatories with a 14W laser for Gemini North (d'Orgeville et al., 2005) ${ }^{[14]}$, a $20 \mathrm{~W}$ laser for Keck I (Lee et al., 2008) ${ }^{[15]}$, a 50W laser for Gemini South (d'Orgeville et al., 2012) ${ }^{[16]}$, and a $5 \mathrm{~W}$ laser for Subaru (Hayano et al., 2010) ${ }^{[17]}$.

While large observatories were able to purchase and install these second-generation lasers, their price remained high, and more importantly their stability was low. Observatories had to invest significant amounts of effort to maintain the laser systems at the specified output power.

\subsection{Third-generation guide star lasers (Fiber lasers)}

The current generation of lasers in use are Fiber lasers. This technology is far simpler to maintain compared to the firstgeneration dye lasers and the second-generation solid-state lasers. That being said it was not until recently that the fiber technology had reached the maturity necessary for the manufacture of these laser. The most used version of these lasers are Raman Fiber Lasers developed by ESO in collaboration with industrial partners. The commercial version of this laser known as the Toptica Sodium star (Enderlein, 2014) ${ }^{[18]}$. This laser has been commissioned at the ESO VLT, the Keck Observatory and the SOR. It has also now been commissioned for the Gemini South telescope and is planned to be deployed at the Gemini North telescope.

For a full and comprehensive review of the history of lasers in astronomy see d'Orgeville and Fetzer $2016^{[19]}$.

\section{OBSERVATION SETUP}

\subsection{Two lasers}

The Gemini South Observatory in 2017 upgraded their second-generation solid-state laser produced by LMCT to a third generation Raman Fiber laser by Toptica photonics. Both of the lasers are located on the telescopes gravity invariant azimuth platform, from there they are sent via a series of free space Beam Transfer Optics (BTO), up along the telescope trusses to the top end ring where a series of polarizing beam splitters divide the beam from one beam into five beams that then propagate across the spider veins and eventually to the laser launch telescope (LLT) that is located behind the 
telescopes secondary mirror. The LLT projects the laser on sky in a five-die shape that is $1 \mathrm{x} 1$ arc-minute square with one spot on each corner and one located in the center.

For the laser upgrade it was decided early in the project that the installation of the new laser would not prevent the operation of the old laser. This means that a method had to be developed to inject the new laser into the BTO without preventing the old laser from being propagated into the BTO, the requirement was also that it would not be possible to propagate both lasers into the $\mathrm{BTO}$ at the same time.

To meet these requirements a new Beam Injection Module (BIM) was developed in house. The BIM has three fold mirrors a half-wave plate and a beam splitter cube. The three fold mirrors are used to send the new laser beam into the BTO with the third fold mirror being deployable. While it is deployed the new laser can propagate into the BTO and the old laser is blocked, and when it is removed the old laser can propagate into the BTO and the new laser is blocked. The half wave plate and the beam splitter cube allow for the new laser to be propagated at low power $(<1 \mathrm{~W})$ into the BTO, this capability is not provided by the laser manufacture. For a full review of the BIM see Chirre et. al $2018^{[20]}$.

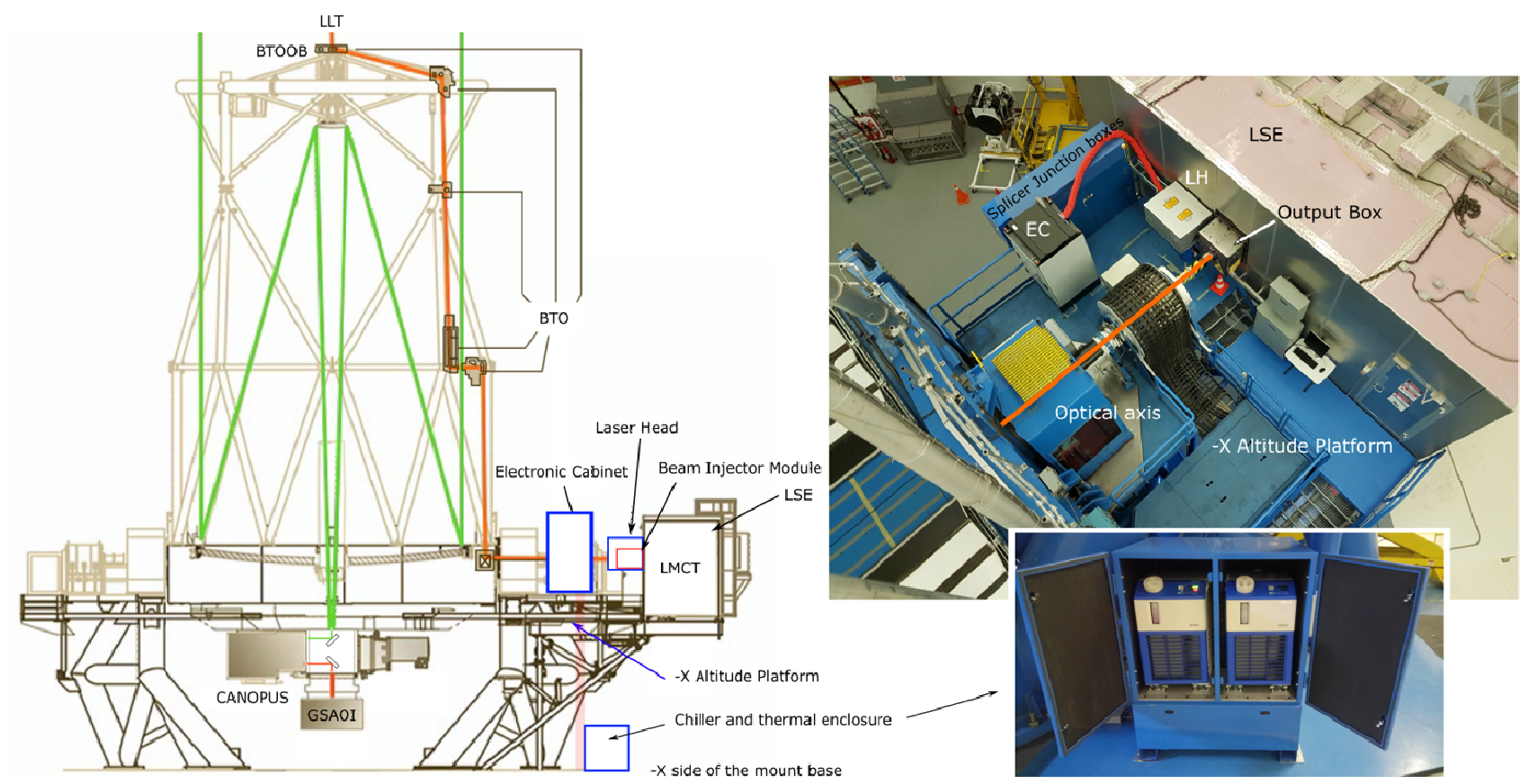

Figure 1. The Gemini south BTO on the left and the new setup with the two lasers on the right. The LSE houses the old secondgeneration laser, while the new third-generation laser is the EC and the LH. The output box houses the BIM.

\section{OBSERVATION SETUP}

\subsection{Observations}

In October of 2017 the Gemini Observatory had its on-sky commissioning run of its new Toptica laser. During 6 nights at the end October the unique opportunity was present to use both laser on sky and do a direct compression of the two lasers on sky performance with only a few minutes of time between the observations. Observations were taken with both the telescopes acquisition camera and the GMOS-S optical imager (Hook, 2004) ${ }^{[21]}$. The observations were done at different telescope elevations and azimuths to fully sample the sky. At each pointing images of the LGS were taking with the acquisition camera, while images with GMOS-S were only taken at Zenith (Elevation $=90$ degrees), in addition to the images of the LGS images were also taken of the detuned laser in order to get the sodium backscatter for subtraction in reduction. The output power of the LMCT laser was $44 \mathrm{~W}$ while the Toptica was $22 \mathrm{~W}$. 


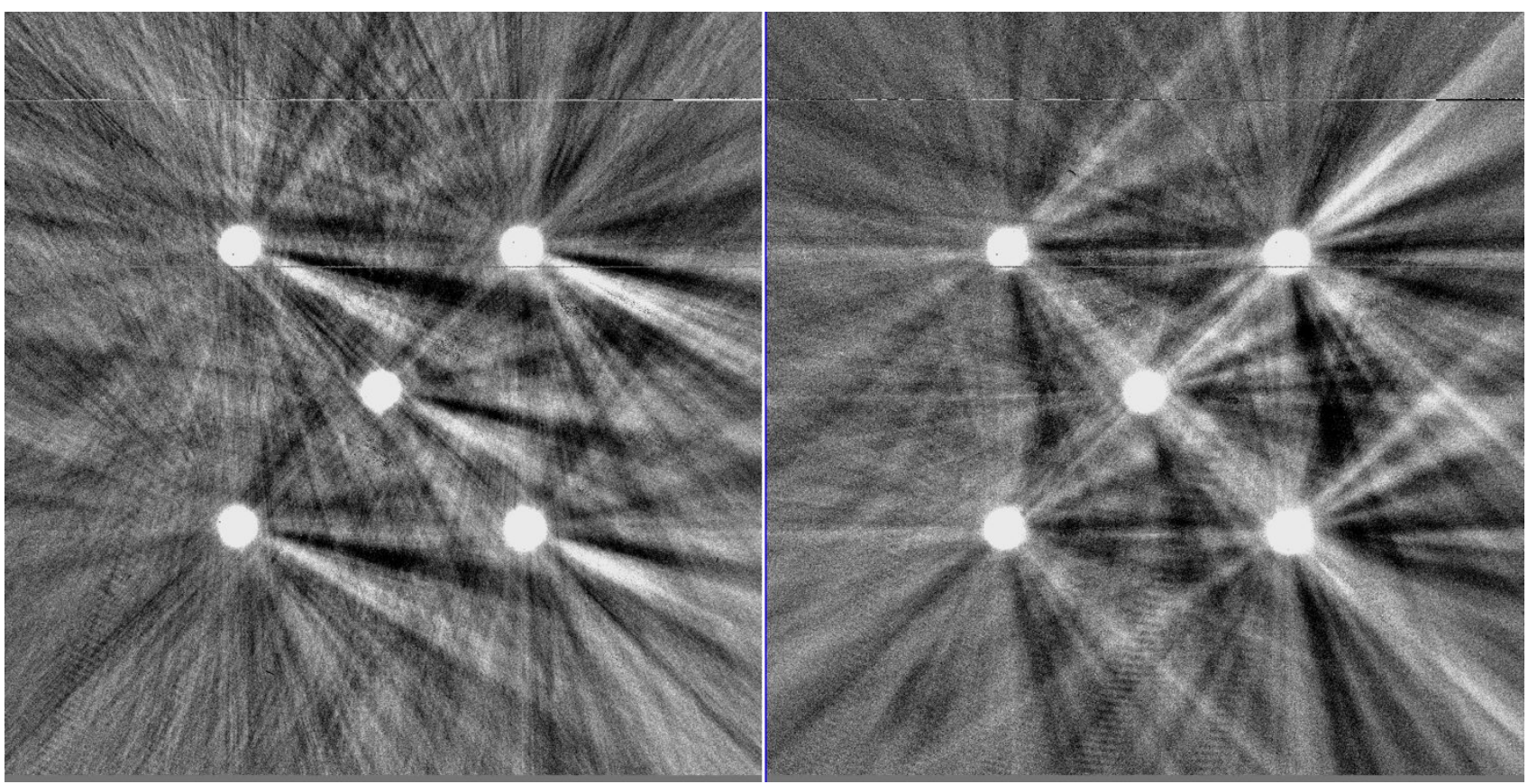

Figure 2: Images of the LGS spots as seen by GMOS-S, the 44W LMCT laser is on the left while the 22W Toptica laser is on the right.

Table 1. Summary of laser observations pointings.

\begin{tabular}{|c|c|}
\hline Telescope Azimuth degrees & Telescope Elevation degrees \\
\hline 0 & 90 \\
\hline 0 & 80 \\
\hline 0 & 70 \\
\hline 0 & 60 \\
\hline 0 & 50 \\
\hline 90 & 80 \\
\hline 90 & 70 \\
\hline 90 & 60 \\
\hline 90 & 50 \\
\hline 180 & 80 \\
\hline 180 & 70 \\
\hline 180 & 60 \\
\hline
\end{tabular}

Proc. of SPIE Vol. $10703107033 \mathrm{~N}-4$ 


\begin{tabular}{|c|c|}
\hline 180 & 50 \\
\hline 270 & 80 \\
\hline 270 & 70 \\
\hline 270 & 60 \\
\hline 270 & 50 \\
\hline
\end{tabular}

\subsection{Photometry}

For each pointing the set of fice images on the acquisition camera were subtracted for the backscatter and then stacked. V-band aperture photometry was taken and a V-band magnitude calculated, the same was done for the GMOS-S data but in r-band. The acquisition camera V-filter has a throughput of $74.2 \%$ at $589 \mathrm{~nm}$ and the r-band filter in GMOS-S has a throughput of $87.5 \%$ at $589 \mathrm{~nm}$. The photometry for each spot was done individually. The magnitudes are reported in table 2 for the zenith pointing in both V-band and r-band.

Table 2. Summary of Zenith V-band and r-band magnitudes

\begin{tabular}{|l|l|l|l|l|}
\hline Spot & $\begin{array}{l}\text { V-Band } \\
\text { Toptica }\end{array}$ & $\begin{array}{l}\text { V-Band } \\
\text { LMCT }\end{array}$ & $\begin{array}{l}\text { r-band } \\
\text { Toptica }\end{array}$ & r-band LMCT \\
\hline Top Left & 10.12 & 9.97 & 9.81 & 9.57 \\
\hline Top Right & 9.79 & 9.62 & 9.38 & 9.17 \\
\hline Center & 10.25 & 10.07 & 9.90 & 9.69 \\
\hline Bottom Left & 10.06 & 9.95 & 9.88 & 9.67 \\
\hline Bottom Right & 9.88 & 9.63 & 9.52 & $9, .25$ \\
\hline
\end{tabular}

Table 3-4 shows how the magnitude changes with different telescope pointings for both lasers using the Top Right spot as it is the brightest, the other spots show a similar pattern.

Table 3. Summary of V-band magnitude for the Toptica laser top right spot, with azimuth on the vertical axis and elevation on the horizontal axis.

\begin{tabular}{|c|r|r|r|r|l|}
\hline & \multicolumn{1}{|c|}{90} & \multicolumn{1}{|c|}{80} & \multicolumn{1}{c|}{70} & \multicolumn{1}{c|}{60} & \multicolumn{1}{c|}{50} \\
\hline 00 & 9.79 & 9.82 & 9.90 & 9.92 & 10.19 \\
\hline 90 & 9.79 & 10.21 & 10.26 & 10.38 & 10.60 \\
\hline 180 & 9.79 & 10.06 & 10.11 & 10.18 & 10.52 \\
\hline 270 & 9.79 & 9.86 & 10.10 & 10.20 & 10.57 \\
\hline
\end{tabular}

Table 4. Summary of V-band magnitude for the LMCT laser top right spot, with azimuth on the vertical axis and elevation on the horizontal axis.

\begin{tabular}{|c|r|r|r|r|l|}
\hline & \multicolumn{1}{|c|}{90} & \multicolumn{1}{|c|}{80} & \multicolumn{1}{c|}{70} & \multicolumn{1}{c|}{60} & \multicolumn{1}{c|}{50} \\
\hline 00 & 9.62 & 9.58 & 9.60 & 9.84 & 10.12 \\
\hline 90 & 9.62 & 9.95 & 10.01 & 10.10 & 10.34 \\
\hline 180 & 9.62 & 9.82 & 9.90 & 9.94 & 10.21 \\
\hline 270 & 9.62 & 9.66 & 9.86 & 9.96 & 10.31 \\
\hline
\end{tabular}


In all cases the LMCT laser is brighter than the Toptica laser by $\sim 0.2$ magnitudes, but as the power of the LMCT is double that of the Toptica laser we can say that the Toptica laser is $60 \%$ more efficient at exciting sodium than the LMCT.

\subsection{Image quality}

The image quality of the LGS was also checked at all pointings. Figure 3 summarizes the image quality measurements.
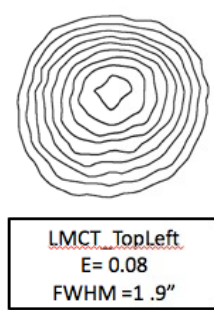

FWHM $=1.9^{\prime \prime}$

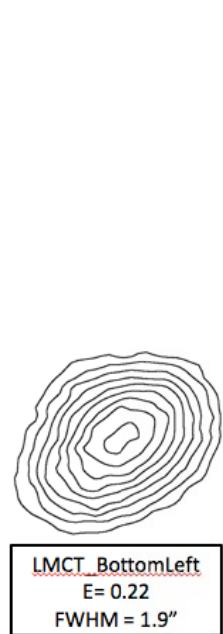

$\mathrm{FWHM}=1.9^{\prime \prime}$
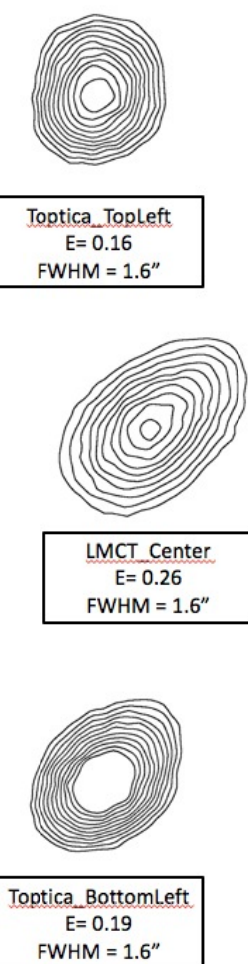
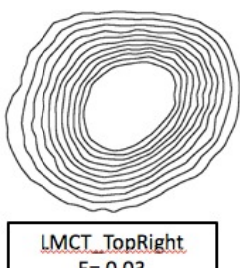

$\mathrm{E}=0.03$

$\mathrm{FWHM}=1.7^{\prime \prime}$
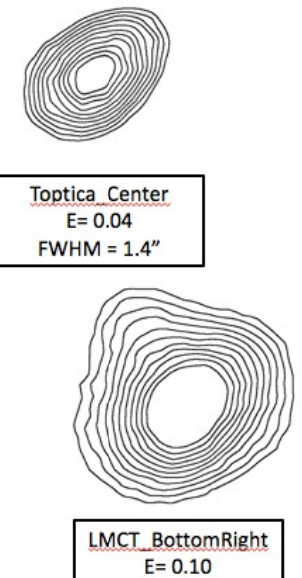

$E=0.10$

FWHM $=1.7$
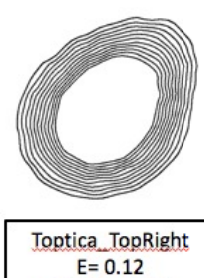

$\mathrm{FWHM}=1.5^{\prime \prime}$

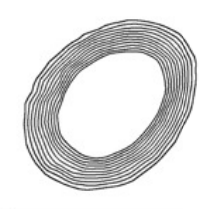

Toptica Bottom Right

$E=0.09$
$F W M=1.4$

Figure 3: Contour plots of the laser spots from the Zenith images with the telescopes acquisition camera. The FWHM in arc seconds is given along with the eliptisity measurements.

The Toptica laser spot is $15 \%$ smaller on sky than the LMCT spot. The noise measurement is proportional to the FHWM, so you get a reduction of the noise measurement. The smaller spot makes the Toptica spots more sensitive to elongation, but this is more than compensated by the smaller spot size.

\section{COMPARISON WITH PREDICTED RETURN}

\subsection{Photometric comparison}

Simulations done by Holzlõhner et al (2009) ${ }^{[22]}$, an internal study done by Rochester Scientific and measurements of sodium retune on Cerro Pachón and Cerro Tololo by Neichel el al $(2013)^{[23]}$, predicted that the output from the Toptica laser should be at least as good as that of a 35W LMCT laser. As our LMCT laser was 44W the prediction from Rochester is consistent with what we see on sky. That being said the Rochester study done using the LGSBloch simulation package was found to overestimate the return of pulsed lasers by a factor of two (Rampy, 2013) ${ }^{[24]}$. This would mean that even at double the power the LMCT laser should have had a lower return, which is not what we saw in the on-sky observations. We have two main ideas to explain this discrepancy, the first being the relatively low laser power sent to sky. As we split the laser beam into five and with the low throughput of the BTO $(50 \%)$, the flux that is propagated to sky is on the order of a few watts, at this power the benefits of the CW Toptica laser over the LMCT CW mode-locked are less. The other explanation is the poor control of polarization in the Gemini BTO. In the photometric data we collected we can see that each spot has a different intensity, this is because the control of the circular polarization is done as a constellation adjustment and not individually for each beam (Araujo, 2016) ${ }^{[25]}$. 


\section{CONCLUSIONS}

Two generations of sodium guide star lasers where used on sky on the same night. The third-generations Raman fiber Toptica laser was $60 \%$ more efficient per watt at exciting sodium than the second-generation solid-sate LMCT laser. This in in agreeance with modeling that shows that the Toptica laser should be more efficient, however it was not as efficient as expected. The reasons for this are likely due to the Gemini environment and not to the laser technologies themselves. The Toptica laser did produce a smaller spot on sky which will lead to a much better single to noise measurement in the AO system. The other added benefit of the Toptica laser is that it is much more reliable and much easier to operate than the LMCT laser.

\section{ACKNOWLEDGMENTS}

The Gemini Observatory is operated by the Association of Universities for Research in Astronomy, Inc., under a cooperative agreement with the NSF on behalf of the Gemini partnership: the National Science Foundation (United States), the National Research Council (Canada), CONICYT (Chile), Ministério da Ciência, Tecnologia e Inovação (Brazil), and Ministerio de Ciencia, Tecnología e Innovación Productiva (Argentina).

\section{REFERENCES}

[1] Foy, R. and Labeyrie, A. "Feasibility of adaptive optics telescope with laser probe," A\&A, 152, L29-L31 (1985)

[2] Thompson, L. A., and Gardner, C. S., "Experiments on laser guide stars at Mauna Kea Observatory for adaptive imaging in astronomy", Nature 328, 229-231 (1987).

[3] Rigaut, F. et al., "Gemini multiconjugate adaptive optics system review - I. Design, trade-offs and integration," MNRAS, 437, 2361 (2014)

[4] Neichel, B. et al., "Gemini multiconjugate adaptive optics system review - II. Commissioning, operation and overall performance," MNRAS, 440, 1002 (2014)

[5] Avicola, K. el al., "Sodium-layer guide-star experimental results", JOSA A 11, 825-831 (1994)

[6] Friedman, H. W., et. al., "Sodium beacon laser system for the Lick Observatory". SPIE 2534 (1995)

[7] Friedman, H. W., et. al., "Design of a laser guide star system for the Keck II Telescope" ESO workshop on laser technology for laser guide star adaptive optics astronomy", ESO 55 (1997)

[8] Rabien, S., et. al., "Design of PARSEC the VLT laser", SPIE 4839, 393 (2003)

[9] Bonaccini Calia. D., et al., "First light of the ESO laser guide star facility", SPIE 6271, 627207 (2006)

[10] Jeys, T. H., "Development of a mesospheric sodium laser beacon for atmospheric adaptive optics", The Lincoln Laboratory Journal 4, 2 (1991)

[11] Dekany, R., et al., "Laser guide star adaptive optics on the 5.1 meter telescope at Palomar Observatory", AMOS (2005)

[12] Denman, C., et al., "Realization of a 50-watt facility-class sodium guidestar pump laser_, SPIE 5707, (2005)

[13] Drummnd, J., el al., "The Sodium LGS Brightness Model over the SOR", AMOS, E67 (2007)

[14] D’Orgeville, C., et al., "Gemini North Laser Guide Star First Light”, AMOS, (2005)

[15] Lee, I., et al., "20W and 50W guidestar lasers systems update for Keck I and Gemini South telescopes", SPIE, 7015 (2008)

[16]D'Orgeville, C., et al., "Gemini South multi-conjugate adaptive optics (GeMS) laser guide star facility on-sky performance results" SPIE, 8447, 84471Q (2012).

[17] Hanyano Y., et al., "Commissioning status of Subaru laser guide adaptive optics system", SPIE 7736, 21 (2010)

[18] Enderlein, M., et al., "Series production of next-generation guide-star lasers at TOPTICA and MPBC," Proc. SPIE 9148, 914807 (2014).

[19] D’Orgeville, C., Fetzer, G. J., "Four Generatios of Sodium Guide Star Lasers for Adaptive Optics in Astronomy and Space Situational Aqareness". SPIE, 9909, 99090R-1 (2016)

[20] Chiree, E. et al., "Switching between two Laser Guide Star Facilities: An overview of the opto0mechanical design for the new laser beam injector at the Gemini South observatory", SPIE this volume (2018)

[21]Hook, I. et al., "The Gemini-North Multiobject Spectrograph: Performance in Imaging, Long-slit, and Multi-Object Spectroscopic Modes", PASP, 116, p. 425-440 (2004)

[22] Holzlöhner, R et al, "Optimization of cw sodium laser guide star efficiency," Astronomy and Astrophysics 510, 20 (2009). 
[23] Neichel, Benoit et al. "Characterization of the sodium layer at Cerro Pachón, and impact on laser guide star performance." Monthly Notices of the Royal Astronomical Society, 3522-3532, (2013).

[24] Rampy, R. "Advancing Adaptive Optics Technology: Laboratory Turbulence Simulation and Optimization of Laser Guide Stars," PhD Dissertation (2013). http://escholarship.org/uc/item/0s8530f2

[25] Araujo, A., et al., "Polarization control optimization of the Gemini South beam transfer optics", SPIE 9148, 91483J9 (2016). 